\title{
Site correlation, anomalous diffusion, and enhancement of the localization length
}

\author{
Paolo Allegrini \\ Center for Nonlinear Science, University of North Texas, P.O. Box 5368, Denton, Texas 76203-5368 \\ Luca Bonci \\ Center for Nonlinear Science, University of North Texas, P.O. Box 5368, Denton, Texas 76203-5368 \\ and Dipartimento di Fisica dell'Universita' di Pisa, Piazza Torricelli 2, 56100, Pisa, Italy \\ Paolo Grigolini \\ Center for Nonlinear Science, University of North Texas, P.O. Box 5368, Denton, Texas 76203-5368; \\ Dipartimento di Fisica dell'Universita' di Pisa, Piazza Torricelli 2, 56100, Pisa, Italy; \\ and Istituto di Biofisica del Consiglio Nazionale delle Ricerche, Via S. Lorenzo 26, 56127 Pisa, Italy \\ Bruce J. West \\ Center for Nonlinear Science, University of North Texas, P.O. Box 5368, Denton, Texas 76203-5368
}

(Received 24 April 1996)

\begin{abstract}
Herein we study the effects on Anderson localization of correlations in the energy distribution of the sites of a tight-binding Hamiltonian. The lattice correlations are introduced by means of classical maps generating anomalous diffusion, that have recently been found to account for the correlated disorder of "biological" lattices. We show that the enhancement of localization length takes place on a much wider band of energies than in the case of the random-dimer model if the random walk on the site energies of the tight-binding Hamiltonian is determined by the joint action of short- and long-range correlations. [S0163-1829(96)08641-9]
\end{abstract}

It has been over a quarter century since Anderson $^{1}$ introduced the concept of localization to describe the spatial properties of an electron wave function on a lattice having random potentials in space. In the last few years the attention of many researchers has focused on the breakdown of this localization caused by the presence of correlations in the random distribution of the Hamiltonian matrix elements. The standard model is the tight-binding Hamiltonian and the correlations are assumed either between diagonal and offdiagonal matrix elements ${ }^{2}$ or among the diagonal ones only, as in the case of the random-dimer model (RDM) of Dunlap, $\mathrm{Wu}$, and Philips. ${ }^{3}$ Heinrichs ${ }^{4}$ studied three simple types of correlated, continuously distributed site energies: a randomdimer model, a random-trimer model, and a random monomer-dimer model, and he found "antilocalization",4 as well as localization and delocalization.

Further light has been recently shed on the correlationinduced delocalization of the RDM by Izrailev, Kottos, and Tsironis $^{5}$ who established a correspondence between the RDM and a classical Hamiltonian map, thereby establishing analytically the existence of transparent states for two values of the energy.

A different approach to introducing the site correlations has been followed by other authors. ${ }^{6,7}$ Crisanti et al. ${ }^{7}$ use a master equation method (MEM) and assume that the site energy is a dichotomous variable with the values $W$ and $-W$. Further, they assume that the probability for the nearest neighbor of a given site to keep the same energy as the given site is $\epsilon$, with $\epsilon \leqslant 1$, whereas the probability of getting an energy with the opposite value of the given site is $1-\epsilon$. This is proven to result in a correlation function for the fluctua- tions of the site energies to decay exponentially with the correlation length: $L(\epsilon)=(-\ln |2 \epsilon-1|)^{-1}$.

More recently, there has been some interest in quasiperiodic lattices, e.g., the Fibonacci lattice ${ }^{8}$ and the Thue-Morse lattice. ${ }^{9}$ In this paper we introduce site correlations by means of a different kind of lattice model, which we conjecture might have some relevance for DNA-protein interaction. ${ }^{10} \mathrm{It}$ has recently been assessed by Allegrini et al. ${ }^{11}$ that the longrange correlation in DNA sequences can be satisfactorily accounted for by adopting as a generator of the sequence a deterministic map recently developed by Leibovitch and Tóth $^{12}$ for different purposes. The Leibovitch and Tóth map (LTM) results in anomalous diffusion and produces the same kind of long-range correlations as the Geisel-NierwetbergZacherl map ${ }^{13}$ (GNZM) used earlier by Tréfan et al. ${ }^{14}$ to study the deterministic approach to anomalous diffusion. However the LTM affords the advantage of supplementing the GNZM long-range properties with a short-range correlation reminiscent of that of the RDM.

The purpose of this paper is to demonstrate the different roles that short- and long-range correlations have on the process of enhancement of the localization length $\lambda$. The arguments are based on the numerical calculations of the Lyapunov exponents, to determine $\lambda$ as a function of the energy, in the same form as that recently adopted by Davids. ${ }^{15}$ We shall compare the effects produced by the LTM and GNZM to a conventional random lattice, uncorrelated at both short and long ranges, which we shall refer to as a fully random lattice (FRL), as well as to the RDM and the MEM.

To begin, let us write the tight-binding Hamiltonian as 


$$
H=\sum_{m} \xi_{m}|m\rangle\left\langle m\left|+V \sum_{m=n \pm 1}\right| n\right\rangle\langle m|,
$$

where for simplicity we assume the condition of nearestneighbor interactions and constant coupling $V$. As far as the distribution of the site energies $\xi_{m}$ is concerned, we assume it to be generated by the deterministic maps presented below.

The explicit form of the GNZM is given by

$$
y_{i+1}=f\left(y_{i}\right),
$$

where

$$
f(y)=\left\{\begin{array}{l}
y+a y^{z} \text { for } 0 \leqslant y \leqslant d, \\
y+a y^{z}-1 \quad \text { for } d<y<1 / 2, \\
y+1-a(1-y)^{z} \text { for } 1 / 2 \leqslant y<1-d, \\
y-a(1-y)^{z} \text { for } 1-d \leqslant y \leqslant 1,
\end{array}\right.
$$

and $d$ is defined implicitly by means of $d+a d^{z}=1$ and $a=2^{z}$. The fluctuating variable $\xi_{m}$ takes the values $+W$ or $-W$, where $W$ defines the noise intensity, and is determined by

$$
\xi_{i}=W\left(\left[2 y_{i}\right]-1\right),
$$

where $[\cdots]$ denotes the integer value.

The explicit form of the LTM, on the other hand, is given by

$$
f(y)=\left\{\begin{array}{l}
y+a y^{z} \text { for } 0 \leqslant y \leqslant d, \\
\frac{1-d-y}{1-2 d} \text { for } d<y<1-d, \\
y-a(1-y)^{z} \text { for } 1-d \leqslant y \leqslant 1,
\end{array}\right.
$$

where $a=(1-2 d)^{1-z}$. The fluctuating variable $\xi_{m}$ is defined as in Eq. (4).

Notice that these maps have been used for a deterministic approach to diffusion, ${ }^{13,14}$ which implies $\xi_{m}$ is a fluctuating function of time. In this case both the mappings produce a diffusive behavior with an increase of the second moment proportional to $t^{4-\mu}$ where $z \equiv \mu /(\mu-1)$. In the forthcoming we shall consider the case with $z=2 \rightarrow \mu=2$, i.e., a superdiffusive case.

In this paper the variable time is replaced by a variable position, denoting the site of the Hamiltonian chain Eq. (1). Thus the time correlations of the fluctuations of the "velocity" $\xi_{m}$ here become space correlations among distinct sites. The waiting time distribution in one of the two states, $+W$ or $-W$, becomes the length distribution of lattice clusters of adjacent sites with the same energy. The main difference between the GNZM and the LTM is that in the latter alternating sequences $\cdots W,-W, W \cdots$ or $\cdots-W, W,-W \cdots$, of any length, are rigorously forbidden, while in the former they are possible. Of course, these alternating sequences are admitted also by the MEM. In the language of the deterministic approach to anomalous diffusion, the LTM is characterized by the property that the waiting time in one of the two states must be greater than 1, whereas both the GNZM and the MEM would admit a waiting time of 1 . With the LTM the minimum waiting time in one of the states is equal to 2 , namely that corre- sponding to the RDM. Sequences of the type $\cdots W$, $W,-W,-W, W, W \cdots$ of the same type as those produced by the RDM, are possible together with sequences of the type $\cdots W, W,-W,-W,-W, W, W \cdots$ and others with still larger waiting times in one of the two states. It is evident therefore that in spite of the fact that both the GNZM and the LTM maps result in the same long-range correlations and in the same anomalous diffusion, as described in Refs. 11 and 14, a striking common property, the LTM can be regarded as a generalization of the RDM, whereas the GNZM is rather a generalization of the FRL. The MEM shares a property with both the FRL and GNZM, namely the possibility of producing the alternating sequences $\cdots W,-W, W$ or $-W, W$, $-W \cdots$. However, the MEM results in a finite correlation length, and produces an infinite correlation length only in the limiting case of ordered lattices, whereas both the GNZM and the LTM result always in sequences with an infinite correlation length.

As mentioned earlier, to establish the localization length of the states as a function of energy we adopt the method of detection of the Lyapunov coefficients in the same form as that of the recent paper by Davids ${ }^{15}$. We build up lattices with 500000 sites, thereby implying that in the case of the LTM and GNZM we make these maps run for an equal number of time steps. Of course, also the random number generators behind the FRL and the MEM are made to run for the same number of sites. The results are illustrated in Fig. 1. Note that in all the figures the horizontal dotted line denotes the sample size, thereby making it possible to establish visually when a state with a finite but very large correlation can be considered as being virtually equivalent to a delocalized state.

From Fig. 1(b) we see that the RDM is characterized by two sharp peaks centered around the two infinitely extended states, whose existence was established with analytical arguments by Izrailev, Kottos and Tsironis. ${ }^{5}$ However, as the spectrum departs from the peaks it tends to recover the behavior corresponding to the FRL. Notice that the RDM curve predicts localization lengths which on the shoulders are smaller than those predicted by the FRL, a property reminiscent of that discovered by Crisanti et al. ${ }^{7}$ (we shall come back to this aspect later on in this paper). In conclusion, the RDM short-range correlation results in two infinitely extended states surrounded by a region of states with extremely large but finite values of $\lambda$. As earlier noted, states with a finite value of $\lambda$ larger than the size of the sample, as far as the conduction properties are concerned are indistinguishable from delocalized states. However, from Fig. 1(b) we see that the band of states with $\lambda$ larger than the sample size, and thus virtually infinite, is relatively sharp. We see from Fig. 1(c) that also the GNZM produces values of $\lambda$ larger than those of the FRL. Furthermore, the band of states with increased localization length is significantly broader than that produced by the RDM. We notice, however, that the largest values of $\lambda$ are still much shorter than the sample size.

Figure 1(d) is devoted to comparing the FRL with the MEM. It has to be pointed out that at $\epsilon=1 / 2$ the MEM is equivalent to the FRL. Correlations are created by making $\epsilon$ depart from this value while keeping it within the interval $[0,1]$. However, the nature of the correlations produced by 

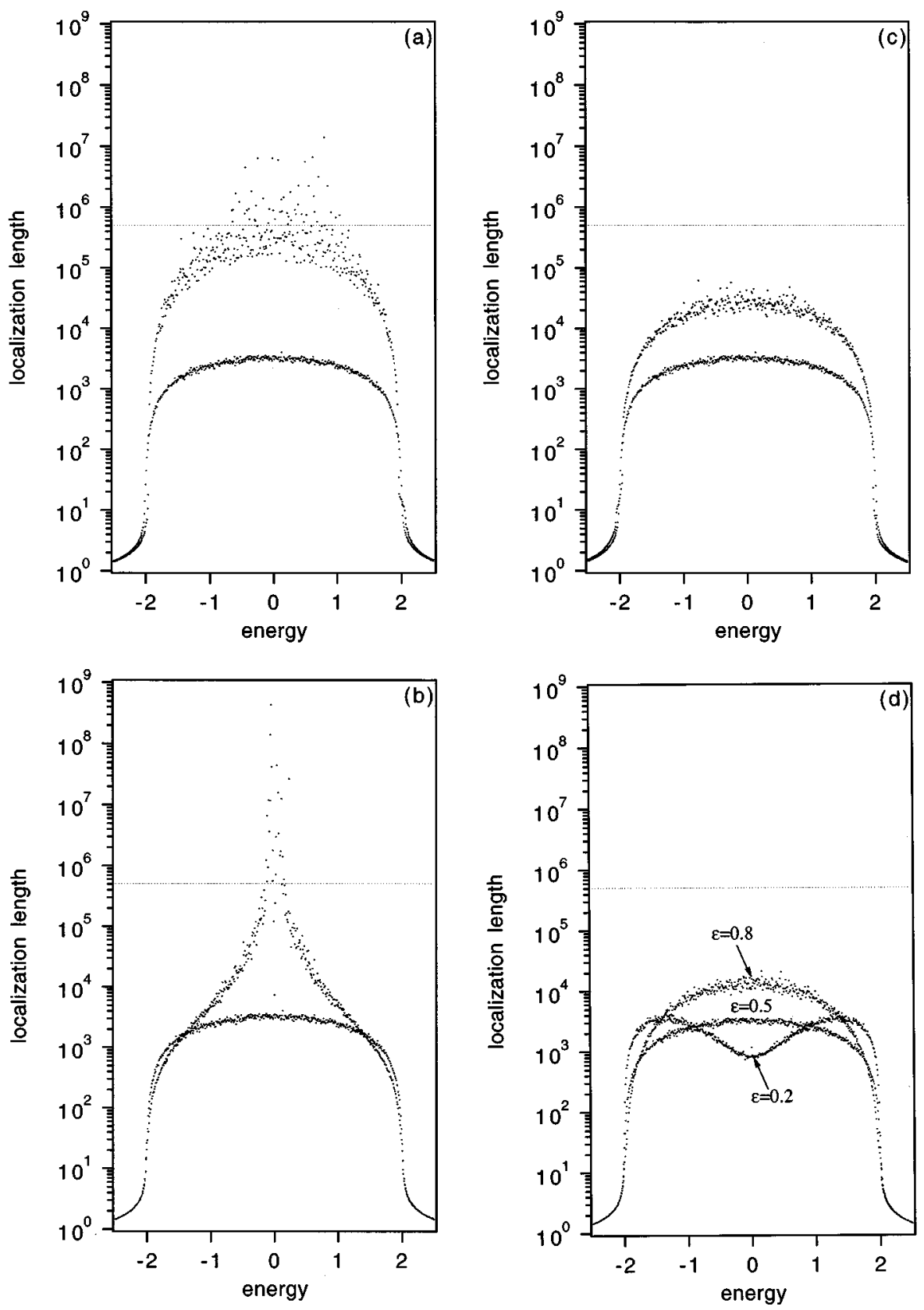

FIG. 1. The localization length $\lambda$ as a function of the energy $E$. In all the four figures we show for comparison with the different cases studied the result of the FRL prediction. (a) the upper curve denotes the prediction of the LTM; (b) the upper curve denotes the prediction of the RDM; (c) the upper curve denotes the prediction of the GNZM; (d) the predictions of the MEM. Note that the curve $\epsilon=0.5$ coincides with the predictions of the FRL.

the region $\epsilon<1 / 2$ is different from that produced by the region $\epsilon>1 / 2$. The limiting case of infinite correlation lengths in the former region is $\epsilon=0$ : it corresponds to infinitely extended alternating sequences and results in a localized state at $E=0$. In the latter region, on the contrary, the limiting case of infinitely extended correlations is $\epsilon=1$, which corresponds to the regular lattice. In accordance with the observations made by Crisanti $e t a l{ }^{7}$, we find that in the former case [see the case $\epsilon=0.2$ of Fig. 1(d)] a sort of hole is created at $E=0$. In the latter case [see the case $\epsilon=0.8$ of Fig. 1(d)] the MEM results in a curve for the localization length as a function of the energy reminiscent of that of the GNZM of Fig. 1(c). We notice also that in the region corresponding to the shoulders of the FRL curve, as mentioned earlier in this note, the MEM produces values of $\lambda$ smaller rather than larger than those of the FRL.

The most interesting result of this note is given by Fig. 1(a), illustrating the predictions of the LTM. We see that the
LTM establishes states with values of $\lambda$ which are orders of magnitude larger than those produced by the FRL. Even remarkable is the fact that this significant enhancement applies to a band of states much broader than that produced by the RDM. We think that the enhancement of $\lambda$ is triggered by the short-range correlations, namely by the fact that, as pointed out earlier, the map of Eq. (5) rigorously forbids the birth of the alternating sequences: $\cdots W,-W, W,-W, W,-W \cdots$. On the other hand, the longrange correlation of the LTM, a property that the LTM shares with the GNZM, gives the band of virtually transparent states the same broad structure as that appearing in Fig. $1(\mathrm{c})$.

We notice that both the LTM and the GNZM are characterized, for our choice $\mu=2$, by an infinite "correlation time." This means that all the statistical observations made on a truncated chain imply large fluctuations of $\lambda$, and consequently of the Lyapunov coefficient. In the case of the 
LTM [Fig. 1(a)] these fluctuations are much stronger than in the case of the GNZM [Fig. 1(c)] because the enhancement of the localization length provoked by the lack of alternating sequences make them comparable to the finite size of the sample. Thus the large fluctuations exceeding the size of the sample in Fig. 1(a) are probably a numerical artifact provoked by the limited statistics available: in the energy region where the enhancement of $\lambda$ is very large a reliable convergence of the Lyapunov coefficients to the correct value is not even allowed by a sample with 500000 sites. However, we trust the indication afforded by the numerical calculation, that the values of $\lambda$ are comparable to the size of the sample.

We stress that we have made numerical checks to assess the effects of abolishing the alternating sequences. In fact the two mappings, Eqs. (3) and (5), produce two realizations of the $\xi_{i}$ sequences which mainly differ on the short-range scale, while both sequences have the same long-range power-law correlation. This can be quantified studying the the mean "laminar region" $L$, namely the mean length of site sequences carrying the same value (either $+W$ or $-W)$. Note that the value $L$ is determined by the map parameters of Eqs. (3) and (5) (Refs. 11-14) or, in our $\mu=2$ case, fixed by the finite length of the sample used. The numerical calculations are made with the LTM resulting in a value for $L$ much larger than that yielded by the GNZM thereby giving the impresssion that the localization length enhancement might be essentially provoked by the increase of this parameter. However, this is not the case. This is proved by changing the parameter $d$ in such a way as to get values of $L$ as small as to that corresponding to the GNZM case illustrated in Fig. 1(c), and still forbidding the birth of alternating sequences. We have proved that changing $L$ of about one order of magnitude does not modify the LTM prediction: the upper curve of Fig. 1(a) remains esssentially unchanged in the scale of this figure. This shows that the enhancement of about one order of magnitude obtained by replacing the GNZM of Fig. 1(c) with the LTM of Fig. 1(a) is mainly due to the fact that the LTM is incompatible with the presence of alternating sequences.

In conclusion, on the basis of our numerical results we are now in a position to establish that the long-range correlations produce a significant increase of $\lambda$ over a broadband of en- ergy values. The MEM in the region of $\epsilon$ leading to the birth of alternating sequences seems to be an exception to this rule. However, by adopting the language of the deterministic approach to diffusion ${ }^{14}$ we remark that a process favoring the birth of alternating sequences would lead to subdiffusion rather than superdiffusion, if any deviation from standard diffusion could be detected. This means an anomalous diffusion slower than Brownian diffusion, rather than faster. We restrict our attention to the case of anomalous diffusion coresponding to processes faster than ordinary Brownian motion. We notice also that the long-range correlation of the MEM for $\epsilon=0.8$ produce a result similar to that of the correlations with infinite correlation length of the GNZM. Thus, we are led to establish this empirical rule: if the correlation function of $\xi$ does not have any oscillation leading to negative values, and its decay is so slow as to lead to either a very large or an infinite correlation length, a broad band of eigenstates with an enhanced value of $\lambda$ is produced. However, this long-range correlation effect by itself would not be so efficient in creating states with exceptionally large values of $\lambda$. States with very large values of $\lambda$ actually are created by short-range properties similar to those of the RDM, where the minimum "waiting time" is 2 . The short-range correlation mechanism, excluding totally the possibility of alternating sequences of any length, brings into existence bands of states with values of $\lambda$ comparable to the sample size. Then the long-range correlations have the further and significant effect of broadening these bands.

This interesting property is made especially transparent by comparing Fig. 1(b), corresponding to the prediction of the RDM, with Fig. 1(a), illustrating the effects produced by the LTM and the main result of this paper. We see that the RDM, as widely discussed in the earlier work of other groups, ${ }^{2,3,5}$ produces a sharp band of states with values of $\lambda$ comparable to the sample size, and consequently physically indistinguishable from the states with infinite localization length. The LTM makes this band of states significantly broader.

The authors warmly thank Dr. Riccardo Farchioni for illuminating discussions on the subject of this paper, and the Office of Naval Research for partial support of this research.
${ }^{1}$ P. W. Anderson, Phys. Rev. 109, 1492 (1958).

${ }^{2}$ D. H. Dunlap, K. Kundu, and P. Philips, Phys. Rev. B 40, 10999 (1989).

${ }^{3}$ D. Dunlap, H.-L. Wu, and P. W. Philips, Phys. Rev. Lett. 65, 88 (1990).

${ }^{4}$ J. Heinrichs, Phys. Rev. B 51, 5699 (1995).

${ }^{5}$ F. M. Izrailev, T. Kottos, and G.P. Tsironis, Phys. Rev. B 52, 3274 (1995).

${ }^{6}$ M. T. Béal-Monod and G. Forgacs, Phys. Rev. B 37, 6646 (1988).

${ }^{7}$ A. Crisanti, G. Paladin, and A. Vulpiani, Phys. Rev. A 39, 6491 (1989).

${ }^{8}$ P. E. de Brito, C.A.A. da Silva, and H.N. Nazareno, Phys. Rev. B 51, 6096 (1995).
${ }^{9}$ G. Y. Oh, C.S. Ryu, and M.H. Lee, Phys. Rev. B 45, 6400 (1992); C.S. Ryu, G.Y. Oh, and M.H. Lee, ibid. 46, 5162 (1992).

${ }^{10}$ J. J. Ladik, Quantum Theory of Polymers as Solids (Plenum, New York, 1987).

${ }^{11}$ P. Allegrini, M. Barbi, P. Grigolini, and B. J. West, Phys. Rev. E 52, 5281 (1995).

${ }^{12}$ L. S. Liebovitch and T.I. Tóth, J. Theor. Biol. 148, 243 (1991).

${ }^{13}$ T.J. Geisel, J. Nierwetberg, and A. Zacherl, Phys. Rev. Lett. 54, 616 (1985)

${ }^{14}$ G. Tréfan, E. Floriani, B. J. West, and P. Grigolini, Phys. Rev. E 50, 2564 (1994).

${ }^{15}$ P. S. Davids, Phys. Rev. B 52, 4146 (1995). 